\title{
Multi-Robot Odor Distribution Mapping in Realistic Time-Variant Conditions*
}

\author{
Ali Marjovi ${ }^{1}$ and Lino Marques ${ }^{2}$
}

\begin{abstract}
This paper tackles the problem of multi-robot odor distribution mapping through time series analysis. Considering the conditions of real world environments where the chemical concentration distribution is patchy, intermittent and time-variant, we propose a method to incorporate the temporal and spatial aspect of sensory data into the problem of odor distribution mapping. Despite the previous works in this field, the method gives more importance to the recent acquired measurements and also to the measurements which have been spatially closer to the place of the sensors (at the time of their acquisition). Real experiments were done in a realistic smallscale controlled environment (designed for systematic olfactory tests), considering up to five real robots and two different navigation algorithms. Experiments show that the generated odor maps are remarkably more accurate than the results of the conventional spatial interpolation method. Studying various spatio-temporal neighborhoods in the time series analysis concluded that a proper definition of the neighborhood (in time and space) provides accurate results in gas distribution mapping.
\end{abstract}

Keywords: Gas/odor distribution mapping, Robotics olfaction, Time series analysis.

\section{INTRODUCTION}

Environmental monitoring, chemical leak detection, pollution monitoring, inspection of landfills, and search and rescue operations are the main applications of gas distribution mapping with mobile robots. Some of these tasks are done in scenarios extremely dangerous for humans, being desirable to use robots instead.

Understanding how the odor molecules disperse through the environment under naturally turbulent flow is the key to design and develop efficient robotic olfactory mapping strategies. Odor patches released by an odor source are mainly transported by the airflow, forming an odor plume. As the plume travels away from the source, it becomes more diluted due to molecular diffusion and turbulence that mixes the odor molecules with clean air [1]. Molecular diffusion is a slow process whose effect on the plume shape and the odor concentration can be neglected. In ventilated indoor or in outdoor environments, the dispersion of odor molecules is dominated by flow turbulence. The odor molecules move downwind due to mean flow velocity $\vec{U}$ while their net motion is almost random, due to small scale turbulence curls. As flow carries odor patches away from the source, the average concentration within a patch decreases and

\footnotetext{
*This work was partially supported by the European project TIRAMISU under grant FP7/SEC/284747.

${ }^{1}$ Ali Marjovi is with DISAL laboratory, EPFL University, Lausanne, Switzerland. ali.marjovi depfl.ch

${ }^{2}$ Lino Marques is with the Institute of Systems and Robotics, University of Coimbra, Portugal. lino@isr.uc.pt
}

simultaneously the average time between successive patches increases, producing concentration intermittency. Several researchers, including [2], reported instantaneous concentration fluctuations with peaks up to three orders of magnitude above the average value. Under these circumstances, a fast chemical sensor located far enough downwind of the odor source can only detect the odor peaks and will measure no odor concentration in most of the time. Fig. 1 presents the nature of an odor plume at various temporal scales.

Turbulent behavior of airflow, lack of smooth odor concentration gradient, patchiness of odor depression, meandering and time variant characteristics of odor plumes imply that in real world conditions, a mobile sensor network able to acquire data spread in space has significant perception advantages in comparison to a single robot that can only measure the concentration at its own place. Multiple robots can spread out in the environment setting up a dynamic spatial sensor network.

The field of mobile robotics olfaction includes a number of main research directions including "odor distribution mapping" and "olfactory search". The "olfactory search" consists of three subproblems; odor plume finding [3], odor plume tracking [4], [5], and odor source declaration [6]. "Odor distribution mapping" is the problem of accurately acquiring and presenting the olfactory sensory data in an environment [7]. In contrast to our previous studies [3], [8] that were mainly focused on olfactory search and environment exploration, this paper tackles the different problem of odor distribution mapping, using time series analysis concepts.

To state the problem, we consider an application in which a group of $N$ individual robots perform a navigation task in an unknown environment contaminated with a chemical volatile. The robots are equipped with the necessary hardware to sense the contaminating chemical. Like in any real-world application, the odor distribution is time variant. The objective is to build a dynamic map of the chemical concentration as well as to determine the region of highest concentration.

This problem has been addressed by several researchers in the past years using different approaches. Hayes et al. [9] aim to localize an odor source through the odor map of the environment. This map is generated averaging raw sensor readings collected by a group of mobile robots performing random walks. Farrell et al. [10] employed hidden Markov methods to estimate the location of odor source based on an odor concentration map. Marques et al. [11] presented a mapping methodology which first estimates the odor concentration by means of a neural network-based 

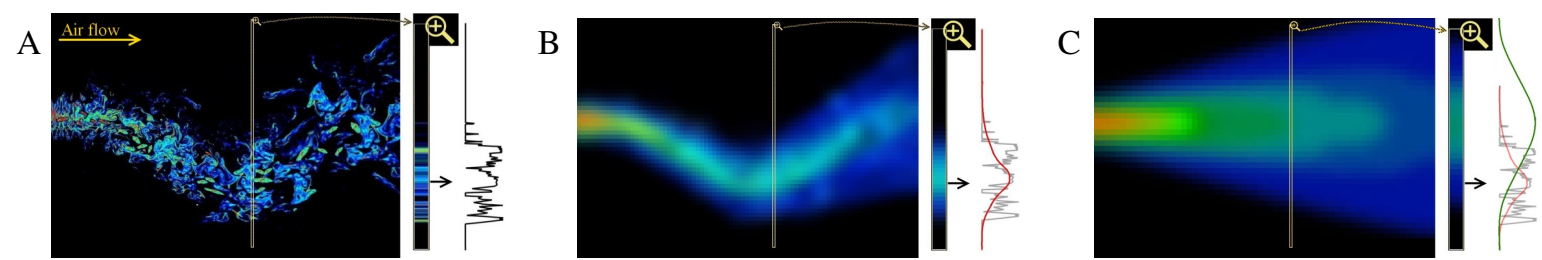

Fig. 1. Odor plume structure in various exposures. A. instantaneous structure (adapted from [2]), B. The spatial average of the odor plume, and C. The time (and spatial) average of the odor plume. The black signal in A shows the instantaneous measurements of a fast gas sensor while moving cross-wind, the red signal in B shows the output of a slow sensor (that acts like a low-pass filter) that moves cross-wind, the green signal in C shows the average of the measurements during a long time period.

regression algorithm and then assimilates the sensed data into an advection-diffusion model by means of a reduced order Kalman filter. Lilienthal and his colleagues have done the most extensive work in this area in the past ten years. Their first works generated a grid map of the odor concentration using a Gaussian density function to model the decreasing likelihood that a particular reading represents the true concentration with respect to the distance from the point of measurement [7]. Later, this method was improved by "Kernel DM+V method", which learns a statistical 2D gas distribution from a sequence of localized gas sensor measurements taking mean and variance of odor concentration both into account. Estimating the variance, this algorithm is able to suggest new measurement locations that may improve the uncertainty of the estimated gas concentration map. A major weakness of this method, and also most the the ones previously mentioned, is assuming stationary conditions for the environment and only using spatial dimensions to weight the map estimates without taking into account the time dimension. It is clear, from the previous description of the characteristics of odor plumes, that this assumption is not valid in real world conditions with turbulent flow. Therefore, a common drawback of the previous methods is that they do not consider the impact of time in the mapping. Asadi et al. [12] provides an exception to the previous approaches being somehow related to this one. They improved "Kernel $\mathrm{DM}+\mathrm{V}$ " method by adding a time-dependency term $\varphi$ which is defined as an exponential function of time, in order to introduce a decreasing importance of measurements with increasing time between measurement and prediction. However, their work considers two phases of the gas distribution mapping independently (one in space domain the other in time domain), first gas concentration grid maps are generated in space domain (using "Kernel DM+V"), then the effect of time is applied to the previously estimated maps. While in our work, these two processes are considered related, since gas distribution is a spatio-temporal phenomenon, and the relating parameters were experimentally investigated and tuned in order to obtain reliable odor maps. It should be mentioned that there are a few studies in sensor networks literature which do consider time but do not take mobility of the sensors into account. The mobility of the robots implies that measured olfactory data during time do not correspond to constant positions in space.

Time series are sets of ordered observations on quantitative characteristics of a phenomenon at equally spaced time points (Brocklebank et al. [13]). Methods based on time series analysis extract meaningful statistics and other characteristics from sequenced data. Prediction and forecasting, classification, regression analysis and signal estimation are the main applications of these methods [14]. We propose a method based on time series analysis to incorporate the temporal and spatial aspect of data into the problem of odor distribution mapping. Since the robots' acquired olfactory data is actually a time series, this paper tackles odor distribution mapping problem through time series analysis approach. The goal is to generate a map for the odor at current time while the area is not fully explored and the actual values of all the points are not available. The common approach is using some kind of regression to estimate values at unobserved positions based on the previous observed data. This method utilizes a weighting function to generate the odor map at time $t$ using not only spatial data at $t$, but also the history of measurements taken at previous time steps. The justification here is that the odor value at a specific point in the exploration area is correlated to the other points in its spatio-temporal neighborhood. Proposing a weighing function which gives more importance to the recent acquired measurements and also to the measurements which have been spatially closer to the place of the sensors (at the time of their acquisition) is the main contribution of this paper.

\section{PROPOSED METHOD}

A solution to deal with intermittency and patchiness of odor dispersal is to use the mean concentration values gathered during the motion of the robots to estimate the local concentration gradient [5]. Applying a low-pass filter on the instantaneous sensor measurements at different places provides a smooth local gradient towards the plume center line and also towards the source (see Fig. 1.B). This will address the problem of dealing with fluctuations and patchiness of odor dispersal. If another low-pass filter is applied to this data during the time, a pseudo-Gaussian plume is obtained whose gradient is towards the source (see Fig. 1.C). This addresses the problem of dealing with plume meandering in real world environments. Therefore, to obtain a correct longterm exposure of the odor dispersion we need to apply two correlated processes to the sensory measurements: (i) spatial interpolation/extrapolation, and (ii) temporal diffusion. This paper uses time series analysis to implement these two processes. Since the robots are spread through space they can cooperatively act as a spatial interpolation/extrapolation filter. 


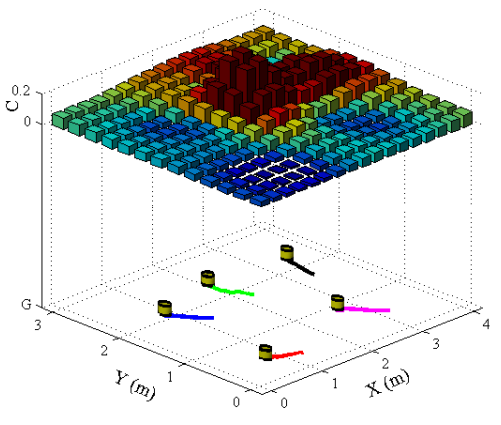

Fig. 2. Robots trajectory and generated odor map in one time snap. Five robots have been moving in a $4 \times 3 \mathrm{~m}^{2}$ arena while logging the odor concentrations. The upper bar-matrix represents the result of the equation (1) on this sensory data.

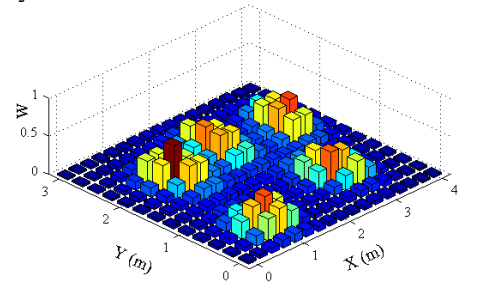

Fig. 3. The current weights calculated by equation 3 while trajectory of the robots were shown in Fig. 2. The places which are close to the trajectory of the robots gain higher weights in the interpolation process.

Moreover the data series gathered by the robots during the time can be analytically overlapped to generate a consistent odor map. The challenge is that the sensory data of different time-snaps cannot be easily overlapped since the data is not observed from fixed locations due to the robots' mobility.

Robots sense the odor concentration with their sensors while they are navigating in the environment. In each short period of time (a time snap) the data gathered from different and known scattered set of points is broad-casted to the neighbors. A multivariate interpolation is needed to estimate the values for the other points of the environment in each time snap. These values are calculated with a weighted average of the values available at the known points. The inverse of the distance to each known point (the proximity value) is used as the weight of effectiveness of the value of the known point in the estimation.

Having $N$ odor sensors and denoting concentration readings as $C_{i}(t), i=1 . . N$ in known positions $\left(x_{1}, y_{1}\right)$, $\left(x_{2}, y_{2}\right), \ldots,\left(x_{N}, y_{N}\right)$ at time $t$, the interpolated value $C\left(x_{j}, y_{j}, t\right)$ at a given point $\left(x_{j}, y_{j}\right)$ is estimated using "inverse distance" weights model [15], as follows:

$$
C\left(x_{j}, y_{j}, t\right)=\frac{\sum_{i=1}^{N} w_{i}\left(x_{j}, y_{j}, t\right) C_{i}(t)}{\sum_{i=1}^{N} w_{i}\left(x_{j}, y_{j}, t\right)}
$$

where weights $w_{i}\left(x_{j}, y_{j}, t\right)$ are given by:

$$
w_{i}\left(x_{j}, y_{j}, t\right)=\frac{1}{\left[d_{t}(i, j)\right]^{p}}
$$

where $d_{t}(i, j)$ is the spatial distance between the locations $\left(x_{i}, y_{i}\right)$ and $\left(x_{j}, y_{j}\right)$ at time $t$ and $p$ is a positive real number, called the power parameter whose default value is 2 .

The odor distribution map in this paper is represented by a uniform grid matrix, so any Cartesian $(\mathrm{x}, \mathrm{y})$ denotes a grid cell with center at (x,y). By calculating the equation (1) for all grid cells, a map is generated that corresponds to the current status of odor distribution in the environment at time $t$. Fig. 2 shows an experimental example of this equation on a real-world data set. In this example, during a five second time window the robots have been moving in the space and logged the odor concentration. Using equation (1) and (2), the upper bar-matrix is generated that represents the odor map of the environment in one time slice. In this example the grid size was set to $0.25 m$ and $p=2$. We denote $W$ as the summation of the weights:

$$
W\left(x_{j}, y_{j}, t\right)=\sum_{i=1}^{N} w_{i}\left(x_{j}, y_{j}, t\right)
$$

Therefore, $W\left(x_{j}, y_{j}, t\right)$ is a factor that implies how close point $(x, y)$ is to the sensed points $\left(x_{i}, y_{i}\right), i=1 . . N$ in an estimation. If a point is far from the sensed positions (robots) the summation of its weights will be a small value. Fig. 3 is an example that shows the normalized summation of weights for estimation of all points of Fig. 2. This figure shows that places closer to the robots' trajectory gain higher weights in the interpolation process. These weights will be later used in the time series analysis process.

The process of estimating $C\left(x_{j}, y_{j}, t\right)$ and $W\left(x_{j}, y_{j}, t\right)$ is done for all time snaps and the result is a series of odor maps during the time. Now the question is how to merge (or overlap) these maps to each other and find a consistent odor distribution map. Fig. 4. (Left) presents a time series generated by implementing this process on a real-world data (more details about the experiments are given in section III).

Using the spatio-temporal estimate (1) we formalize the problem of generating a comprehensive map of odor distribution as a prediction problem where the values of the target time series are forecasted using not only previous values of the series and summaries of its temporal dynamics, but also with spatio-temporal indicators that summarize the dynamics of the series within the neighborhood. We tackle this problem by the assumption that odor concentration depend not only on the recent past values at the same location but also on nearby locations. The first question to be addressed is how to describe the behavior of the time series within the neighborhood of the target location. The spatio-temporal neighborhood is the region which includes all points within a space-time distance margin. We define a function to calculate the distance between any two points in the space-time dimension. Let $i$ and $j$ be two points in spacetime (i.e. two measurements or estimates $C_{i}(t), C_{j}(t)$ ). We define the spatio-temporal distance between $i$ and $j$ in a similar way to [16]:

$$
\operatorname{Dist}_{i, j, t}=d_{t}(i, j) \times \alpha+T_{i, j} \times(1-\alpha)
$$



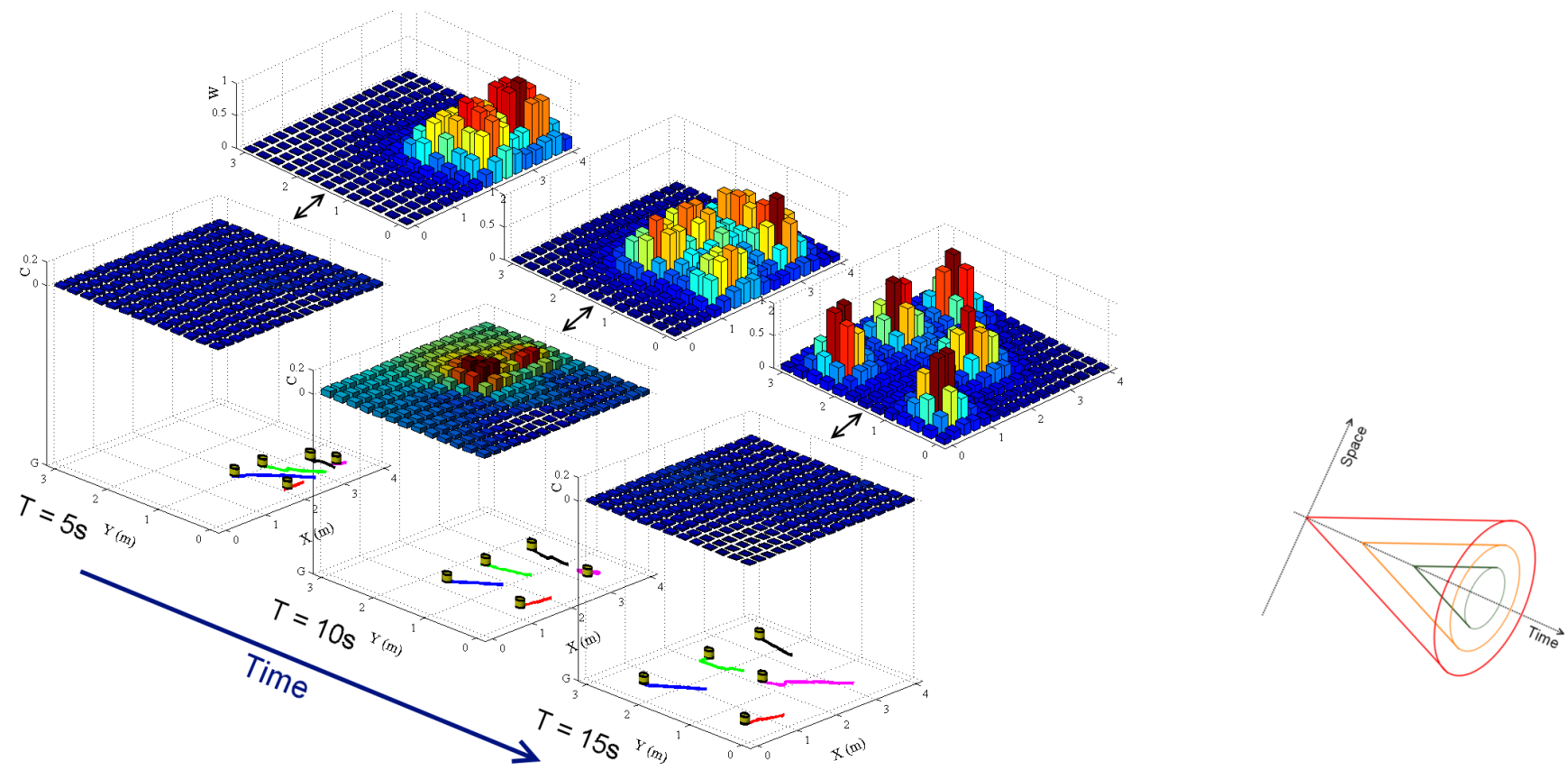

Fig. 4. Left: the generated time series of odor distribution maps and the current weights. Right: spatio-temporal neighborhoods with different sizes.

where $T_{i, j}$ is the time distance between two data points $t_{i}$ and $t_{j}$, and $\alpha$ is weighing factor $(0<\alpha<1)$ between time and geographical distances that are assumed to be normalized. The time distance is simply the absolute difference between the two time tags in some adequate time unit (e.g., seconds).

Based on the defined spatio-temporal distance between two data points, the spatio-temporal neighborhood of a point $o$ is defined:

$$
N_{o}^{\beta}=\left\{j \in D: \text { Dist }_{o, j, t}<\beta\right\}
$$

where D is the available spatio-temporal data set. Given the above definitions, the spatio-temporal neighborhood of a point can be seen as a cone shape within space-time dimensions. Different settings for $\alpha$ and $\beta$ lead to cones of different sizes as shown in Fig. 4. (Right). These cones represent which past values may influence the future value of the time series at the central location. Increasing the size of the cone will increase neighborhood size.

Assuming that in each time snap $\mathrm{C}(\mathrm{x}, \mathrm{y}, \mathrm{t})$ is measured as the interpolated odor concentration at time $t$, we define the spatio-temporal predicted concentration at time $t$ as:

$$
S\left(x_{j}, y_{j}, t\right)=\frac{\sum_{k=1}^{t-1} \sum_{i \in N_{j}} C\left(x_{i}, y_{i}, k\right) \times W\left(x_{i}, y_{i}, k\right) \times U(i, j, k)}{\sum_{k=1}^{t-1} \sum_{i \in N_{j}} W\left(x_{i}, y_{i}, k\right) \times U(i, j, k)}
$$

where $N_{j}$ stands for the spatio-temporal neighborhood $N_{j}^{\beta}$ (in 5), and we define $U(i, j, k)$ weights as:

$$
U(i, j, k)=\frac{1}{\text { Dist }_{i, j, k}}
$$

Equation (6) implies that the estimated predicted concentration at time $t$ is dependent on two factors:
- the distance in time and space $\left(D_{\left.i s t_{i, j, k}\right)}\right.$ of all the previous acquisitions inside $N_{j}$,

- the gain weights calculated in space based on the trajectory of the robots in each time snap $(W)$ from time $=1$ to $t-1$.

Therefore, for any given grid cell, those measured values $(C)$ which are closer to the trajectory of the robots have higher impact on the estimated final value of the cell $(S)$. This proximity in time and space provided by equation (4) leads to significant results in odor mapping.

In each time snap, the value of $\mathrm{S}$ for all grid cells is calculated based on the sensor readings values (C) in all previous time snaps (up to $t-1$ ) inside the defined spatiotemporal neighborhood. The grid map generated by the values of $S$ is an estimate of the future time snap of the odor concentration (at $t$ ) and also include all the information of the history of sensor readings. We call this map as the overall odor distribution map in this paper. The actually measured odor concentration (C) at time $t$ can be used as the groundtruth to evaluate the prediction result in S.

Parameters $\alpha$ and $\beta$ for the spatio-temporal neighborhood (equations 4 and 5) need to be set regarding the environmental elements. These parameters depend on the characteristics of odor distribution in the environment. In the next section we study the values of $\alpha$ and $\beta$ in a set of real world experiments.

\section{EXPERIMENTS}

The proposed approach for odor distribution mapping was evaluated with a set of experiments in realistic conditions.

1) The robots: A set of $\mathrm{LSE}_{\mathrm{mini}}{ }^{1}$ robots were developed at our laboratory in ISR-UC based on the $2 \mathrm{WD}$ miniQ ${ }^{2}$

\footnotetext{
${ }^{1} \mathrm{http}: / /$ lse.isr.uc.pt/news/lseminiqrobot

${ }^{2}$ http://www.dfrobot.com
} 


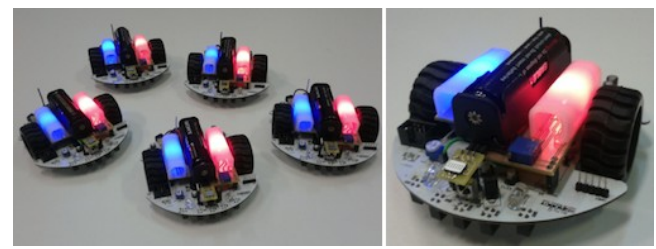

Fig. 5. The developed LSE MiniQ robots containing gas sensors, XBee modules and LEDs.

platform. The LSE miniQ (shown in Fig. 5) communicates with a host computer using XBee. ${ }^{3}$ The host computer runs ROS $^{4}$ programs (nodes) to control the robots. Each robot is controlled by an individual ROS node. A single computer can run several ROS nodes, i.e., a swarm of LSE miniQs. Each robot contains an e2v MiCS-5524 gas sensor to detect volatile organic compounds. Two LEDs (one blue and one red) are installed on top of the robots in order to visually track the robots by a camera mounted at the ceiling. The SwisTrack ${ }^{5}$ software is used to track and to localize the robots. This system provides all the requirements to easily run and to monitor various experiments on a swarm of robots inside the testbed.

2) Realistic small scale environment: A key asset to carry out systematic olfactory experiments is to have an environment under controlled fluid dynamic conditions. In an uncontrolled environment, usually the performance of the algorithms is not quantitatively measurable due to unknown changes in the environmental conditions (e.g. flow speed). For this reason, a small scale realistic environment specifically for olfactory robotics experiments has been developed in our laboratory. The odor distribution mapping method was tested in this realistic environment shown in Fig. 6. This arena, with $3 \times 4 \mathrm{~m}^{2}$ area by 0.5 meters height, has controlled ventilation through a manifold that extracts air from the testing environment through a honeycomb mesh integrated into one of the walls. The opposite surface of the environment contains a similar mesh that allows the entrance of clean air flowing through the environment. The wind speed was set to $0.7 \pm 0.1 \mathrm{~m} / \mathrm{s}$ during the tests. The ceiling of this testbed is covered by a sheet of transparent Plexiglas to be visible from the outside. A controlled acetone gas source using bubblers is pumped to arbitrary places of the environment through a set of PolyVinyl Chloride (PVC) tubes. The acetone release rate was about 0.01 to $0.03 \mathrm{~g} / \mathrm{s}$ during the tests.

3) The process of the experiments: Experiments were done with two different algorithms for robots' navigation:

- decentralized asynchronous particle swarm optimization (DAPSO) (detailed in [17]),

- diagonal cross-wind line up sweep (detailed in [3]).

In different tests, four or five robots were released in one corner of the testbed and the odor distribution maps were estimated. Fig. 7 presents one of the real world experiment.

\footnotetext{
${ }^{3}$ http://www.digi.com/xbee/

${ }^{4}$ http://www.ros.org

${ }^{5}$ http://www.ros.org/wiki/swistrack
}

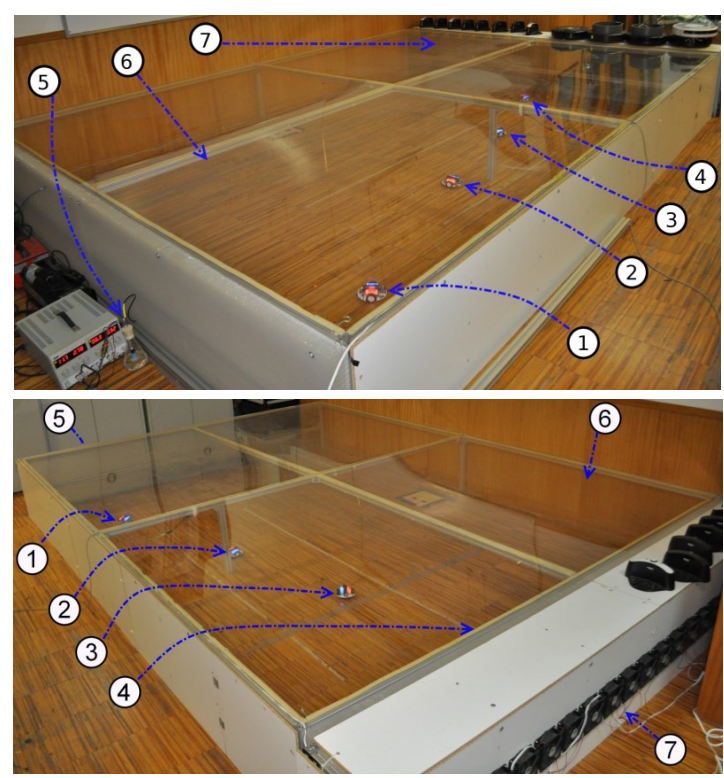

Fig. 6. The realistic testbed environment viewing from two different angles. 1,2,3,4: robots, 5: odor source (Acetone release bubbler), 6: transparent Plexiglas ceiling, 7: ventilation system

The left column shows the ROS environment and the realist test bed and the robots, while the right column presents the corresponding time series process. We considered the following two positions for the odor source release: $(2.5,1.2) \mathrm{m}$ or $(1.7,2) \mathrm{m}$. Each particular test was repeated two times (once having the source at $(2.5,1.2)$ and another time at $(1.7,2))$.

The odor concentration data logged during each navigation algorithm was fed to the mapping method. We used a five second window size to split the data and generate a time series. The target variable was to predict the odor concentration at time $\mathrm{t}+5 \mathrm{~s}$. We assume the following linear distance function in time dimension: $T_{i, j}=0.1 \times\left|t_{j}-t_{i}\right|$ while $t_{i}$ and $t_{j}$ are calculated in seconds. This means that in equation (4) each 10 seconds implies 1 distance unit.

The proposed mapping method was compared to the conventional spatial interpolation, having five different sets of values for $\alpha$ and $\beta$. As we have seen the configuration of neighborhoods are cones defined by Equation (4). By setting various values to $\alpha$ and $\beta$, different neighborhood cones are defined and we can study the way that the available past data affects the forecast of the target variable. For instance, $\alpha=0.5$ and $\beta=0.5$ defines a cone with maximum radius of $1 \mathrm{~m}$ at the current time snap and height of 10 seconds, i.e., a neighborhood that for the current time uses points that are at most $1 \mathrm{~m}$ away from the target location, and goes back in time at most 10 seconds. We denote this neighborhood region by $[1 \mathrm{~m}, 10 \mathrm{~s}]$ in this paper. Using this terminology and considering $\beta=0.5$, we can describe the six set of parameters for the mapping process as follows:

1) Pure spatial interpolation (no history considered),

2) $\alpha=0.4$, neighborhood: $[1.25 \mathrm{~m}, 8.33 \mathrm{sec}]$,

3) $\alpha=0.6$, neighborhood: $[0.83 \mathrm{~m}, 12.5 \mathrm{sec}]$,

4) $\alpha=0.8$, neighborhood: $[0.625 \mathrm{~m}, 25 \mathrm{sec}]$, 


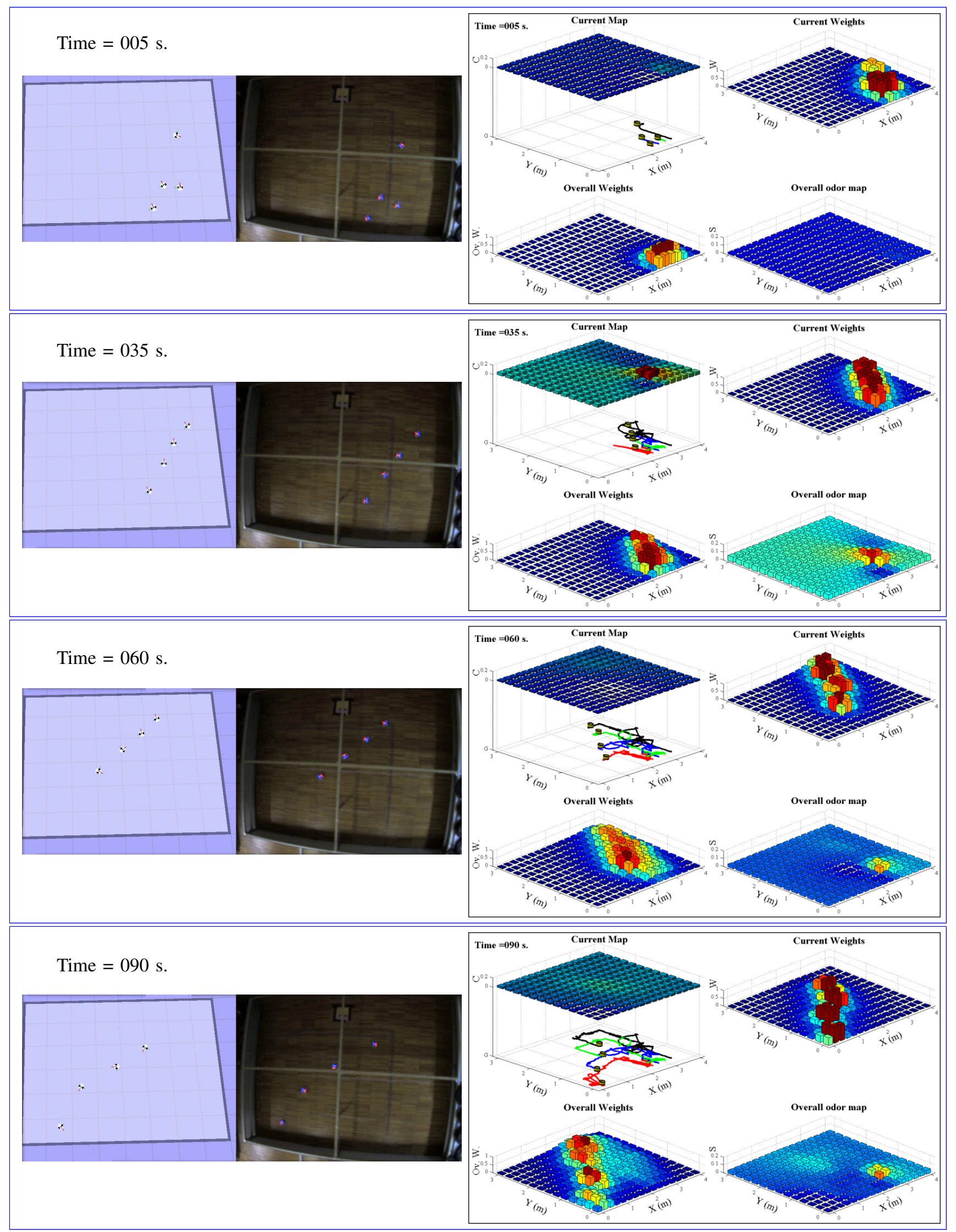

Fig. 7. A real world experiment. The left column shows the ROS environment and the realist test bed and the robots, while the right column presents the corresponding time series process. Each frame shows the trajectory of the robots, the interpolated current odor map $(\mathrm{C})$, the current weights of interpolation, the overall weights and the overall odor map. It can be seen that the overall odor maps does not fluctuate very much and has a peak always around the odor source position at $(2.5,1.2) \mathrm{m}$. The difference between the overall weights to the current weights is more significant after a while. 

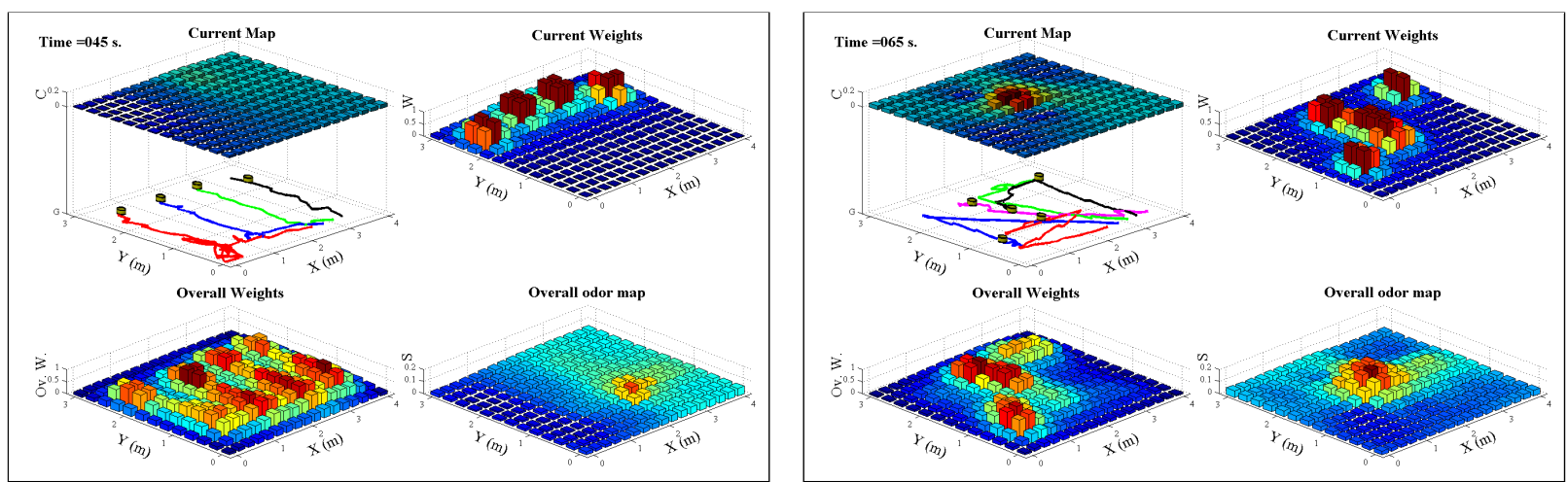

Fig. 8. The generated odor maps during two different experiments. Left: running cross-wind line up navigation algorithm with four robots when the odor was released at $(2.5,1.2) \mathrm{m}$. Right: running DAPSO navigation algorithm with five robots when the odor source was located at $(1.7,2) \mathrm{m}$.

5) $\alpha=0.9$, neighborhood: $[0.55 \mathrm{~m}, 50 \mathrm{sec}]$,

6) $\alpha=0.95$, neighborhood: $[0.52 \mathrm{~m}, 250 \mathrm{sec}]$.

Increasing $\alpha$ leads to expanding the neighborhood cone in time, i.e., considering more historical data to generate the map. The predictions of the different trials were evaluated using the mean absolute error (MAE):

$$
M A E=\frac{1}{N} \sum_{i=1}^{N}\left|S\left(x_{i}, y_{i}, t\right)-C\left(x_{i}, y_{i}, t\right)\right|
$$

where $S\left(x_{i}, y_{i}, t\right)$ is the predicted odor concentration value (calculated by equation 6 based on the time series of time $=$ 1 to $t-1$ ) for a true later experimentally measured value of $C\left(x_{i}, y_{i}, t\right)$ at time $t$. This means that the $\mathrm{C}$ measured in each time snap $t$ is actually ground-truth for evaluating the prediction estimation in $\mathrm{S}$ that is the result of processing the previous time snaps (time $=1$ to $t-1$ ).

At the beginning of an experiment, enough data is not available for prediction, but after a short period of time (and gathering some odor concentration data) the mapping/forecasting process starts. Therefore, we have calculated the MAE for each time step after 30 seconds from starting an experiments.

4) Experimental Results and Discussion: Fig. 9 and Fig. 10 summarize the results of all experiments when the odor source was located at $(1.7,2) \mathrm{m}$. These figures present the mean absolute error (MAE) of all considered variants of the experiments. These charts show that the proposed time series approach performed always better than conventional spatial interpolation mapping in these experiments. Moreover, when there is a big change in the gathered environmental data, the MAE increases suddenly, however, in the proposed method the error decreases again after a few time steps (while in the spatial interpolation the error remains constant most of the times). When the odor source was at $(2.5,1.2)$, the results were similar, so due to brevity we only show results of one setup.

To evaluate the effect of $\alpha$ on the results we calculate the average of MAEs for all the time steps. Fig. 10 presents the normalized mean absolute error for the whole experiments. This figure shows that $\alpha=0.9$ provides more accurate prediction, i.e., more accurate odor distribution maps are
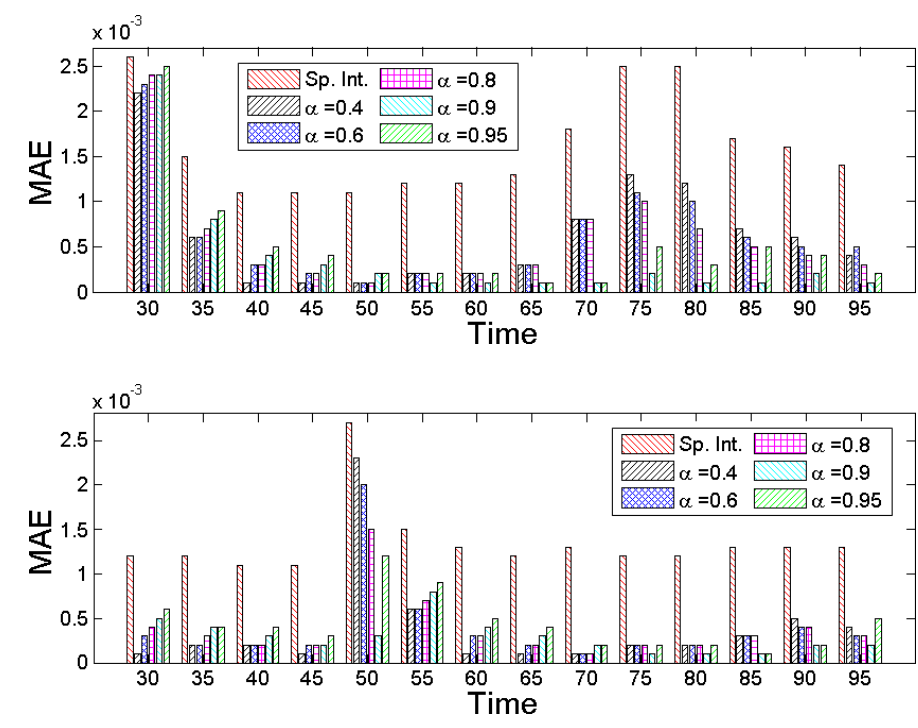

Fig. 9. The mean absolute error calculated in each time step considering (i) pure spatial interpolation, (ii) different values for $\alpha$. (A) running crosswind line up navigation (see Fig. 8.(left)). (B) running DAPSO navigation algorithm (see Fig. 8.(right)).

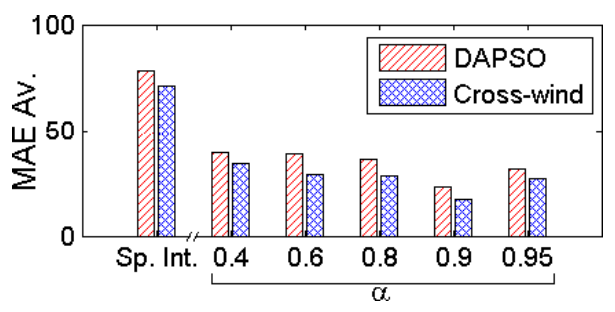

Fig. 10. The normalized overall averaged mean absolute error during the experiments considering various values for $\alpha$. When $\alpha=0.9$ (spatiotemporal neighborhood: $[0.55 \mathrm{~m}, 50 \mathrm{sec}])$ the MAE is minimized.

generated in both navigational algorithms. In other words, when the spatio-temporal neighborhood is $[0.55 \mathrm{~m}, 50 \mathrm{sec}]$ the time series approach provides the best results independent from the navigational algorithm. The point is that the best found neighborhoods are bounded in time and also in space. This results approve that for an accurate odor distribution map, time and space both must be properly incorporated in the mapping method. It is clear that the spatio-temporal neighborhood is dependent on the environmental conditions. 
In addition to the mentioned numerical results, several remarkable observations from the real world experiments can be summarized as follows:

Peak at the location of odor source: The resultant odor distribution maps generated by our method always have peaks at the odor source, despite the pure spatial interpolation method (the left-top frames in the figures 7 and 8). Hence this method can be beneficial to odor source declaration problem.

Gradient towards the source: The gradient of odor distribution maps is [in most of the times and in most of the places] towards the odor source. This implies that this mapping approach will lead to a promising solution for the problem of "odor plume tracking".

Presenting the dynamics of the odor source: Although the generated maps are stable, still if there is any change in the environment or in the odor source, the maps show the changes and are dynamic.

Providing a validity metric for the maps: The overall weight maps (the left-down frames in figures 7 and 8) provide a useful metric to present the degree of certainty and validity of the odor map in each grid cell. The places that the robots have recently visited have higher weights while the places where the robots have not visited [recently] have lower values. These maps can be used in the navigation algorithm of the robots in order to automatically send the robots to the regions with less recent information.

\section{CONCLUSIONS}

The odor mapping method proposed in this paper uses time series analysis to incorporate the temporal and spatial aspect of sensory data to generate overall consistent odor maps. By defining a function for calculating spatio-temporal distance between two points, the method gives more importance to the recent acquired measurements and also to the measurements which have been spatially closer to the place of the sensors (at the time of acquisition). Real world experiments were done in a realistic environment using up to five real robots running DAPSO and "cross-wind line up" navigational algorithms.

The maps generated by the proposed method were compared to the results of the conventional spatial interpolation method. The mean absolute errors in the generated maps of the proposed method were remarkably lower than the errors in the conventional spatial interpolation method. Additionally, through experimental results we determined the near-optimal values of the parameters of the spatiotemporal neighborhood for the considered environment. The best found neighborhoods were bounded in time and also in space. These determined neighborhoods were equal for both navigation algorithms. This implies that the proposed time series approach and the spatio-temporal neighborhoods are independent from the navigational algorithm. The maps generated by this approach are stable which at the same time dynamically present the changes in the odor distribution. Moreover, the peak in the generated maps are mostly located at the real position of the odor source. This mapping approach can be beneficial to address other problems of olfactory robotics namely "odor source declaration" and "odor plume tracking".

\section{REFERENCES}

[1] P. Roberts and D. Webster, Turbulent diffusion. ASCE Press, Reston, Virginia, 2002.

[2] J. Crimaldi, M. Wiley, and J. Koseff, "The relationship between mean and instantaneous structure in turbulent passive scalar plumes," Journal of Turbulence, vol. 3, no. 14, pp. 1-24, 2002.

[3] A. Marjovi and L. Marques, "Optimal spatial formation of swarm robotic gas sensors in odor plume finding," Autonomous Robots, vol. 35, pp. 93-109, 2013.

[4] W. Li, J. Farrell, and R. Cardé, "Tracking of fluid-advected odor plumes: strategies inspired by insect orientation to pheromone," Adaptive Behavior, vol. 9, no. 3-4, p. 143, 2001.

[5] L. Marques, U. Nunes, and A. Almeida, "Olfaction-based mobile robot navigation," Thin Solid Films, vol. 418, no. 1, pp. 51-58, 2002.

[6] G. Cabrita and L. Marques, "Divergence-based odor source declaration," in Control Conference (ASCC), 2013 9th Asian. IEEE, 2013.

[7] A. Lilienthal and T. Duckett, "Building gas concentration gridmaps with a mobile robot," Robotics and Autonomous Systems, vol. 48, no. 1 , pp. 3-16, 2004

[8] A. Marjovi and L. Marques, "Optimal swarm formation for odor plume finding," IEEE Trans. on Cybernetics, 2014.

[9] A. Hayes, A. Martinoli, and R. Goodman, "Distributed odor source localization," IEEE Sensors Journal, vol. 2, no. 3, pp. 260-271, 2002.

[10] J. A. Farrell, S. Pang, and W. Li, "Plume mapping via hidden markov methods," Systems, Man, and Cybernetics, Part B: Cybernetics, IEEE Transactions on, vol. 33, no. 6, pp. 850-863, 2003.

[11] L. Marques, A. Martins, and A. T. de Almeida, "Environmental monitoring with mobile robots," in IEEE/RSJ Int. Conf. on Intelligent Robots and Systems, 2005, pp. 3624-3629.

[12] S. Asadi, M. Reggente, C. Stachniss, C. Plagemann, and A. J. Lilienthal, "Statistical gas distribution modelling using kernel methods," Intelligent Systems for Machine Olfaction: Tools and Methodologies, pp. 153-179, 2011.

[13] J. C. Brocklebank and D. A. Dickey, SAS for forecasting time series, 2nd ed. SAS Institute, 2003.

[14] L. L. Scharf and C. Demeure, Statistical signal processing: detection, estimation, and time series analysis. Addison-Wesley Publishing Company, 1991, vol. 1.

[15] D. Shepard, "A two-dimensional interpolation function for irregularlyspaced data," in Proc. ACM National Conf., 1968, pp. 517-524.

[16] J.-R. Hwang, H.-Y. Kang, and K.-J. Li, "Searching for similar trajectories on road networks using spatio-temporal similarity," in Advances in Databases and Information Systems, 2006, pp. 282-295.

[17] M. Turduev, G. Cabrita, M. Kırtay, V. Gazi, and L. Marques, "Experimental studies on chemical concentration map building by a multirobot system using bio-inspired algorithms," Autonomous Agents and Multi-Agent Systems, vol. 28, no. 1, pp. 72-100, 2014. 